\title{
The role of social comparison for maximizers and satisficers: Wanting the best or wanting to be the best?
}

\author{
Kimberlee Weaver ${ }^{\mathrm{a}, *}$, Kim Daniloski $^{\mathrm{a}, 1}$, Norbert Schwarz ${ }^{\mathrm{b}, \mathrm{c}}$, Keenan Cottone ${ }^{\mathrm{d}}$ \\ ${ }^{a}$ Pamplin College of Business, Virginia Tech, 2016 Pamplin Hall, Blacksburg, VA 24061, USA \\ ${ }^{\mathrm{b}}$ Department of Psychology, University of Southern California, Los Angeles, CA 90089-1061, USA \\ ${ }^{c}$ Marshall School of Business, University of Southern California, Los Angeles, 90089-0443, USA \\ ${ }^{\mathrm{d}}$ Department of Psychology, University of Michigan, Ann Arbor, MI 48109-1048
}

Received 16 October 2012; received in revised form 20 September 2013; accepted 9 October 2014

Available online 18 October 2014

\begin{abstract}
Consumers chose between options that paired either an objectively inferior good with high relative standing (Your laptop is rated 60/100 in quality; others' laptops are rated 50/100) or an objectively superior good with low relative standing (Your laptop is rated 80/100 in quality; others' laptops are rated 95/100). Decision makers who try to make the "best" decision, known as maximizers (Schwartz et al., 2002), pursued relative standing more than decision makers who are satisfied with outcomes that are "good enough" (known as satisficers). That is, maximizers were more likely than satisficers to choose objectively inferior products when they were associated with higher relative standing. Subsequent analyses investigating decisions across time showed that maximizers' interest in relative standing persisted even when the nature of the tradeoff was made overt, suggesting it is a conscious aspect of the maximizer identity. Overall, results suggest that the maximizer self concept is more complex than has been previously assumed - they are focused on relative outcomes in addition to absolute outcomes.
\end{abstract}

(C) 2014 Society for Consumer Psychology. Published by Elsevier Inc. All rights reserved.

Keywords: Consumer decision making; Maximizing; Satisficing; Consumer identity processes; Consumer competition

Imagine that you are given a choice to live in one of two possible worlds. In World A, you drive a car that is a medium quality brand rated $7 / 10$ in parts and performance by Automotive Weekly. Nearly all of your acquaintances' cars are luxury models rated $9 / 10$. In World B, you drive a car that is a fair quality brand, rated $6 / 10$ in parts and performance by Automotive Weekly. Nearly all of your acquaintances' cars are low quality models rated $4 / 10$. If given the choice, would you prefer to live in World A or World B?

\footnotetext{
* Corresponding author.

E-mail addresses: kdweaver@vt.edu (K. Weaver),kimdaniloski@vt.edu (K. Daniloski), norbert.schwarz@usc.edu (N. Schwarz), kcottone@umich.edu (K. Cottone).

${ }^{1}$ Present address: Kania School of Management, University of Scranton, 800 Linden St., Scranton, PA 18510, USA.
}

The focus of our current analysis concerns the conflict that arises between the quality of an item and the relative standing that item confers on its owner as compared to other consumers. Often in the marketplace quality and relative standing are confounded (e.g., high quality signals exclusivity), and the pursuit of the two is not in conflict. However, quality and relative standing are empirically separable constructs and are also distinguishable in many important real world consumer contexts. Quality and relative standing are separated, for instance, in the case of imitation goods, since internal quality is low, but the appearance of quality, and thus the good's relative standing, is high. The literature would suggest that one set of consumers (maximizers) are exhaustive in their search for the best product, while another group of consumers (satisficers) are satisfied with products and outcomes that meet a minimum threshold of acceptability and are thus "good enough." However, as seen above, the best can mean different things. A consumer looking for the best could be 
interested in the best quality product or in the product that gives her the best standing relative to other consumers.

In the current analysis, we use tradeoffs of the type described above to examine a question that past work has not considered: whether maximizers and satisficers as defined by the well-known individual differences scale developed by Schwartz et al. (2002) not only differ in their levels of aspiration for a given standard, but whether they can also differ in the type of standard they choose to pursue in a given consumer choice situation - absolute standards (i.e., product quality) or relative standards (i.e., positional standing). In examining this question we bridge two literatures, one on maximizing and satisficing in decision making and one on relative and absolute choices, which has been studied in the decision making, psychology and consumer behavior literatures (e.g., Easterlin, 1974; Huguet et al., 2009; Zhou \& Soman, 2003).

\section{The maximizer/satisficer distinction}

In an important and influential paper, Barry Schwartz et al. (2002) demonstrated that consumers vary in how much they try to optimize decisions in everyday choice contexts (see also de Bruin, Parker, \& Fischhoff, 2007; Iyengar, Wells, \& Schwartz, 2006; Nenkov, Morrin, Ward, Schwartz, \& Hulland, 2008; Parker, de Bruin, \& Fischhoff, 2007; Swan, 1969; Wright, 1975; Zeelenberg \& Pieters, 2007). Maximizers aspire to acquire the best products and services. Satisficers, on the other hand, are satisfied with products and services that are good enough - those that meet a minimum threshold of acceptability. Thus, while a maximizer may spend hours going from store to store trying on jeans before she finds the best fit, the best style, and the best price, as soon as a satisficer discovers a pair that is good enough, she will purchase them and stop shopping.

Past work utilizing this scale has largely focused on identifying outcomes and decision strategies that are associated with the maximizing decision making style. As would be expected theoretically, this work has shown that, relative to satisficers, maximizers take more time when making decisions (Chowdhury, Ratneshwar, \& Mohanty, 2009; Nenkov et al., 2008; Schwartz et al., 2002), include a larger number of alternatives in their consideration set (Iyengar et al., 2006; Nenkov et al., 2008; Schwartz et al., 2002), exhibit a greater tendency to browse in online shopping environments (Chowdhury et al., 2009), do more background research prior to making choices (Iyengar et al., 2006; Nenkov et al., 2008), and like to choose from a larger array (Dar-Nimrod, Rawn, Lehman, \& Schwartz, 2009). In one prototypical demonstration, maximizers reported being more willing than satisficers to drive an additional 20 minutes to shop at a superstore that allowed them to choose from a larger product array than was available at their local store (Dar-Nimrod et al., 2009).

However, how does a maximizer decide what is best? Generally speaking there are two types of standards that consumers can use when judging what is "best": absolute standards and relative standards (e.g., Easterlin, 1974; Huguet et al., 2009; Zhou \& Soman, 2003). Past work looking at individual differences in the maximization construct has assumed that the primary-indeed "definitional" - goal of maximizers is to achieve absolute standards: the best outcomes in an objective sense. In contrast to this assumption, our major hypothesis in the current paper is that the maximizer self concept is more complex than has been surmised in previous work and that it also includes an aspect of the self that emphasizes relative standards - being the best. If so, then when forced to choose between the two, maximizers may actually be more willing than satisficers to forgo the absolute best under some conditions. We predicted that satisficers, on the other hand, will see little appeal in the relative best and thus, in situations pitting the relative and absolute best against each other, will be more interested in pursuing the absolute best than maximizers. We detail our rationale for this prediction in the next section.

\section{Do maximizers emphasize relative standards more than satisficers?}

One observation from past work that is consistent with the possibility that maximizers may focus on relative standards more than satisficers is the fact that maximizers pay more attention to information about their relative position than satisficers (Schwartz et al., 2002, studies 2 and 3; Polman, 2010). In one demonstration, participants unscrambled anagrams alongside a confederate who was either faster or slower than they were. While satisficers were unaffected by the confederate's performance, maximizers used it as a standard of comparison. They felt worse and rated their own ability as poorer when the confederate was faster than they were (Schwartz et al., 2002, study 3). Maximizers also report engaging in more consumer-related social comparison both before and after making purchases than do satisficers (Schwartz et al., 2002, study 2), suggesting that they are interested in obtaining information about the consumer possessions of others.

However, while it is clear that maximizers are more influenced by and interested in those around them, past work has left the answer to the question of why maximizers are interested in social comparison ambiguous. One possibility is that maximizers always pursue the objective best, and others can provide information about what that best outcome is in an absolute sense. For instance, since college students do not know the statistical distribution of anagram solving ability, knowing the performance of a peer doing the same task can provide valuable information about what an objectively good performance might look like. This possibility of social comparison for the sake of gathering information about the objective standard is articulated by Schwartz (2004), '[w]hile, in theory, 'the best' is an ideal that exists independent of what other people have, in practice, determining the best is so difficult that people fall back on comparisons with others" (p. 199).

However, another possibility can also explain the same pattern of data - a possibility that has not been considered by past work. To the degree that maximizers are hard-driving, competitive people who go to great lengths to obtain the best in life, rather than being single-mindedly concerned with pursuing 
absolute quality, the maximizer identity may actually have different aspects that get activated in different contexts. On the one hand, it may incorporate the goal of being a discerning individual, which leads maximizers to relentlessly pursue objective quality. However, on the other hand, in situations where social rivalry is salient, maximizers' competitive spirit may make salient a potentially competing goal — that of being a "winner," which may lead them to search for the best option in a relative sense, in order to be better than other people rather than to obtain the best absolute outcome. If so, then what may have at first glance appeared to be social comparison in order to obtain objective information about what constitutes a good product or performance may have instead been the manifestation of a distinct facet of the maximizer self image: the desire to be the best in a relative sense. Unfortunately, design characteristics of the existing studies in the literature make it impossible to determine which goal or "best" (i.e., absolute or relative) maximizers are trying to attain through their focus on social comparison.

In the present research, we take a deeper look at maximizers' choices to examine whether they are single-minded in their pursuit of objective quality as has been assumed in previous work, or instead whether they maximize on different goals in different situations - absolute quality when it is salient and relative standing when it is salient. We test this question first by investigating whether maximizers will expend more effort than satisficers to obtain the absolute best as well as obtain the relative best (in situations where the opposite standard is not a consideration) or instead whether maximizers are only more willing to expend effort in cases where absolute outcomes are at stake. Results were consistent with the multifaceted view-maximizers expended effort to achieve both types of outcomes. We then investigate, across a wide variety of consumer products and services, what maximizers and satisficers choose when they are forced to make tradeoffs between those two goals (absolute quality and relative standing). Results again demonstrate the importance of relative standards to maximizers by showing that, contrary to the "definitional" assumption made by previous work, maximizers are more likely than satisficers to choose objectively inferior products, when those products are paired with a higher relative social position (World B in the opening example). Subsequent studies investigate the motivation underlying maximizers' greater preference for positional standing relative to satisficers by examining whether their choices vary as a function of whether the products are publicly visible or not. We test this in three different ways, by empirically manipulating the visibility of the product (study 3 ), by using products that naturally vary in their public visibility (e.g., brand of car vs. brand of pajamas; study 4), and by assessing maximizers' and satisficers' preferences for imitation goods, i.e., goods of lower quality that are designed to have the outside appearance of higher quality goods (study 4). If maximizers' emphasis on social comparison reflects a concern for information about the absolute standards as suggested by past work (Iyengar et al., 2006; Schwartz et al., 2002), then maximizers should choose the same option regardless of whether the outcome of their decision will be publicly visible or not. In contrast, if maximizers are also motivated by social rivalry, then there should be an interaction between the type of decision maker (maximizer vs. satisficer) and the decision context (public vs. private). When the two motivations are juxtaposed, maximizers' competitive nature may lead them to prefer relative standing to absolute quality more than satisficers, unless the situation is private and no one will know about their superiority.

Overall, our results suggest that rather than having a single "definitional" goal of aspiring to obtain the objectively best outcomes that are the best in an objective sense, maximizers have dual goals or identities that can be activated in different contexts - in some situations their choices reflect the goal of being a discerning and quality-seeking consumer, the primary motivation that has been emphasized in past work; however, in other contexts a distinct goal emerges that can under some conditions be shown to contradict the first - the goal of being a "winner" or better relative to others.

\section{Study 1: Single minded or multiple goals?}

Are maximizers specifically concerned with maximizing absolute quality or is an interest in relative standing also a part of the maximizer identity? Past work on the maximizer/satisficer construct has focused on situations where objective quality is at stake and has showed that maximizers expend more effort than satisficers in order to obtain that goal. For instance, in one demonstration, maximizers were more willing than satisficers to complete an additional survey when doing so would allow them to choose a chocolate from a choice set of 36 rather than a choice set of 6 (Dar-Nimrod et al., 2009). In another demonstration, maximizers were more willing than satisficers to drive a long distance to an ice cream parlor that had 200 flavors rather than 20. While it is clear that maximizers are willing to commit effort in order to increase their chance of securing an objectively superior outcome, will the same process apply when the goal is relative standing?

If maximizers are specifically focused on obtaining the highest quality item in an absolute sense, then they should only choose to exert extra effort in contexts where that goal is salient. On the other hand, if the maximizer self concept also includes the concept of being the best, then when social position is a salient goal they should maximize on it too. To test whether maximizers single-mindedly pursue the absolute best or instead are interested in maximizing multiple goals, study 1 presented participants with two types of contexts (i) quality contexts where quality is relevant and relative position is not (e.g., the quality of a dinner that will be consumed alone) and (ii) relative position contexts where relative standing is relevant but absolute quality is not (e.g., winning a contest where the only prize is status). For each type of scenario, participants' willingness to expend effort to obtain a higher level of the motivation (i.e., a better quality dinner; winning the competition) was assessed.

\section{Method}

\section{Participants}

Two hundred and seven participants from an online panel completed this experiment in exchange for payment. Two 
failed to answer the maximization scale, leaving 205 for analysis.

\section{Quality versus relative position scenarios}

Participants saw four scenarios, two that focused specifically on situations where quality was relevant and relative standing was not and two that focused specifically on situations where relative standing was relevant and quality was not. For instance, one of the quality scenarios read as follows:

Imagine that you need a pair of long underwear for an upcoming skiing trip. There is a pair that you can purchase right away that would be fine. There is also a better quality pair on sale for the same price, but to get it you would have to walk all the way to the other side of the mall to a different store. How likely would you be to purchase the pair that is on sale? $(1=$ not at all likely; $7=$ very likely $)$

And one of the relative position scenarios read as follows:

Imagine that your friends gather to play a heated scrabble tournament every month. How hard would you work to win? $(1=$ not at all hard; $7=$ very hard $)$

\section{Maximization scale}

Participants then completed the 13-item maximization scale (Schwartz et al., 2002), which assesses the degree to which individuals try to optimize when making choices (e.g., "When I watch TV, I channel surf, often scanning through the available options even while attempting to watch one program;" "When shopping I have a hard time finding clothing that I really love" $(\alpha=.66$ for the scale)). Each question was measured on a 7-point Likert scale ( $1=$ strongly disagree; $7=$ strongly agree $)$. The more (less) participants agree with the items, the more likely they are to be maximizers (satisficers). Participants' average maximization score was a $4.22(\mathrm{SD}=.77)$ and the median score in the sample was a 4.23. After completing the maximization scale, participants reported demographic information and were thanked for their participation.

\section{Results}

Analyses showed that participants' maximization scores were positively correlated with their willingness to expend effort for both the quality $(r(205)=.16, p<.05)$ and the status $(r(205)=.13, p=.06)$ scenarios. These results suggest that rather than being solely concerned with achieving the objectively best outcomes, the maximizer self concept is multifaceted - maximizers are willing to expend effort both to obtain absolute quality and also to obtain status when these two goals are not directly opposed to each other. Satisficers, on the other hand, are less willing to expend extra effort in securing either quality or status when those goals are presented separately.

\section{Design of experiments $2-4$}

Study 1 showed that maximizers are driven to maximize on both objective quality and on relative standing when the two outcomes are not in opposition to each other. In studies 2-4 we investigate what maximizers and satisficers choose when those two standards are pitted against each other as competing motivations. In particular, we were interested in examining whether maximizers' interest in their relative social position will ever override their desire for quality, the goal that previous work has considered to be "definitional" to the maximizer construct. Participants made a series of choices that required tradeoffs between the absolute quality of a good and the owner's positional standing, as illustrated in our opening example. In each case, one alternative paired a higher absolute standing with a lower relative standing, whereas the other alternative paired a lower absolute standing with a higher relative one. Following Solnick and Hemenway (2005), we refer to the choice of a higher absolute standing at the expense of a lower relative standing as an absolute choice; conversely, we refer to the choice of a higher relative standing at the expense of a lower absolute standing as a positional choice. In terms of the opening example, choosing World A over World B is an absolute choice, whereas choosing World B over World A is a positional choice.

\section{Study 2: Absolute versus positional choices}

Consumers saw 29 tradeoff scenarios encompassing a wide variety of domains including consumer products (e.g., UV protection of sunglasses; MP3 player sound quality), experiences (e.g., length of spring break trip; number of invites to formals), personal attributes (e.g., personal physical shape), and professional status dimensions (e.g., number of job offers; grades on exams). For each scenario, participants chose whether they would prefer an absolute or a positional option.

In addition to examining maximizers' and satisficers' overall preferences for the two types of options, the large number of tradeoff scenarios allowed us to investigate an additional question of theoretical interest - whether the motivation behind maximizers' and satisficers' preferences for one type of option over the other is explicit and conscious or instead whether it is implicit and below participants' conscious awareness. That is, while the specific nature of the tradeoffs - that consumers must choose between quality and status - is present in each scenario, the full implications of what choosing one option over another signifies likely are not evident to participants in the first several choices. However, as participants complete more and more trials, it becomes very clear. After multiple repeated trials, for instance, the choice of a positional option likely starts signaling to the self "I am the type of person who would rather take a lower quality item in order to be better than others." Given these differences in the explicitness of the implications of the tradeoffs for the self over time, an interesting question is whether maximizers' and satisficers' choices of one type of option over another will vary across the repeated trials. If the proportion of absolute versus positional choices they make remains stable over time, it would suggest that the implications of those choices are consistent with participants' conscious identities and compatible with how participants see themselves. On the other hand, if participants' choices of one type of 
alternative, such as positional options, diminish over time, this pattern would suggest that that type of choice is not identity-relevant since conscious awareness of its implications leads consumers to move away from choosing that option.

\section{Method}

\section{Participants}

Fifty-eight undergraduates ( $41 \%$ male) at a large, public, Southeastern university completed this experiment and several unrelated questionnaires as part of an extra credit assignment in their introduction to marketing course.

\section{Positional versus absolute choices}

Participants read the following instructions (adapted from Solnick \& Hemenway, 2005):

Nearly all choices in life involve tradeoffs. In the questions that follow, two different states of the world are presented. You are asked to take a few moments to carefully consider both options and pick which of the two you would prefer. The questions are independent. For each question, pick either the first option or the second option. There are no right or wrong answers; we are looking for your own personal choices.

The computer then presented participants with 29 choice scenarios one at a time in a random order (see Appendix A for examples). For each choice domain, two possible states of the world were offered and participants chose the one they preferred. One state of the world always represented a better absolute outcome paired with a lower positional standing, and the other always represented a lower absolute outcome paired with a better positional standing. For example, the question involving the luxury level of car read as follows:

A Your car rates 5 out of 10 points on a luxury scale; others' cars rate 3 out of 10. [Selection indicative of positional choice]

B Your car rates 7 out of 10 points on a luxury scale; others' cars rate 9 out of 10. [Selection indicative of absolute choice]

The order of options was randomized such that 14 of the 29 scenarios presented the option indicative of a positional choice first, while the other 15 scenarios presented the option indicative of an absolute choice first. We created a composite variable to represent the average number of times consumers chose an absolute versus a positional choice option across the 29 scenarios. Absolute choices were coded as " 0 " and positional choices were coded as " 1 ," meaning the composite variable could range from 0 (chose absolute for all 29 items) to 1 (chose positional for all 29 items).

\section{Maximization scale}

After completing the choice task, participants completed the 13-item maximization scale (Schwartz et al., 2002) $(\alpha=.70$ for the scale). Participants' average maximization score was a 4.36 $(\mathrm{SD}=.77)$ and the median score in the sample was a 4.42 . Participants then reported demographic information and were thanked and debriefed.

Results

We predicted that when relative and absolute outcomes are placed in opposition to each other, maximizers would emphasize relative standards more than satisficers. A regression using the composite variable representing the average number of times participants chose an absolute versus a positional option as the dependent variable and consumers' maximization scores as the independent variable showed a significant positive relationship, such that the higher consumers' maximization scores, the more likely they were to make a positional choice across the 29 choice domains, preferring a higher relative standing even when it came with a lower absolute outcome $(\beta=.46, t=3.88, p<.001)$. This relationship was the same regardless of the reference group investigated ("others": $B=$ $.41, t=3.34, p<.01$; friends: $B=.38, t=3.09, p<.01$, and peers/acquaintances: $B=.30, t=2.34, p<.05$ ). Thus, consistent with predictions, maximizers appear to be more concerned with relative standing than satisficers, even when that relative standing comes at the cost of objective quality.

The statistical analyses described above were performed using consumers' maximization scores as a continuous variable. However, for ease of visual presentation, Table 1 presents the percentage of maximizers (defined as those with maximization scores above the median of our sample) and satisficers (defined as those with maximization scores below the median of our sample) who made a positional choice for each of the choice domains. Out of the 29 contexts studied, maximizers chose positionally more than satisficers in 23 , while satisficers chose more positionally than maximizers in only six; none of the six reversals were significant (exam grades: $\beta=-.42$, Wald $=.51, p=.47$; poker winnings: $\beta=$ .01 , Wald $=.001, p=.97$; Facebook friends: $\beta=.16$, Wald $=$ $.15, p=.70$; rooms in house: $\beta=-.19$, Wald $=.29, p=.59$; eating out: $\beta=.24$, Wald $=.48, p=.49$; going out: $\beta=.63$, Wald $=2.79 p=.10$ ).

\section{Identity processes: Examining choices over time}

Is maximizers' interest in positional standing a conscious and explicit part of the maximizer identity - a conscious way of thinking about the self-or instead is it driven by a more implicit motivation of which they are not consciously aware? To test this, we divided the 29 choice domains into five groups based on the order in which they had been presented to participants (i.e., the first six, the second six, and so on). Results showed that while satisficers' tendency to choose positionally diminished over time (\% choosing positional option: Trials 1-6: 33\%; Trials 7-12: 30\%; Trials 13-18: 24\%; Trials 19-24: 22\%; Trials 25-29: 20\%), maximizers' interest in positional options remained stable (\% choosing positional option: Trials 1-6: 39\%; Trials 7-12: 36\%; Trials 13-18: 37\%; Trials 19-24: 38\%; Trials 25-29: 33\%). This 
Table 1

Study 2: Percentage of maximizers (subjects above the median of the sample on maximization score) and satisficers (subjects below the median of the sample on maximization score) choosing the positional option in each choice domain.

\begin{tabular}{|c|c|c|c|}
\hline & Maximizer & Satisficer & $\begin{array}{l}\text { Gap } \\
\text { (Max - Sat) }\end{array}$ \\
\hline Luxuriousness of your car & 41 & 7 & 34 \\
\hline Quality of your car & 45 & 14 & 31 \\
\hline Quality of your sweater & 41 & 21 & 21 \\
\hline Number of job offers & 28 & 10 & 17 \\
\hline Level of your personal education & 45 & 28 & 17 \\
\hline Yearly income & 31 & 14 & 17 \\
\hline $\begin{array}{l}\text { Chance of getting a job two months } \\
\text { after graduation }\end{array}$ & 31 & 14 & 17 \\
\hline Number of invites to formals & 48 & 31 & 17 \\
\hline Personal physical shape & 31 & 17 & 14 \\
\hline Sound quality of your MP3 player & 55 & 41 & 14 \\
\hline Time spent studying for test & 31 & 21 & 10 \\
\hline Quality of beer you are drinking & 24 & 14 & 10 \\
\hline $\begin{array}{l}\text { Number of days you are in Florida } \\
\text { for spring break }\end{array}$ & 10 & 0 & 10 \\
\hline UV protection of your sunglasses & 48 & 41 & 7 \\
\hline Frequency of sex per month & 21 & 14 & 7 \\
\hline Time spent training for athletics & 31 & 24 & 7 \\
\hline $\begin{array}{l}\text { Number of bids received for a } \\
\text { fraternity/sorority }\end{array}$ & 31 & 24 & 7 \\
\hline Number of invites to parties & 48 & 45 & 3 \\
\hline Frequency of going to movies & 45 & 41 & 3 \\
\hline Quality of suit for interview & 45 & 41 & 3 \\
\hline Number of DVDs owned & 21 & 21 & 0 \\
\hline What row your football seats are in & 17 & 17 & 0 \\
\hline Quality of spring break destination & 34 & 34 & 0 \\
\hline Grades on exams & 7 & 10 & -3 \\
\hline Poker winnings & 28 & 31 & -3 \\
\hline Number of Facebook friends & 17 & 24 & -7 \\
\hline Number of rooms in house & 41 & 48 & -7 \\
\hline Frequency of eating out per month & 38 & 52 & -14 \\
\hline Frequency of going out per week & 21 & 55 & -34 \\
\hline
\end{tabular}

provides evidence consistent with the idea that an interest in relative standing is an overt part of the maximizer identity. See Table 2 for the pattern of results.

\section{Discussion}

Study 2 shows that, rather than always striving for the objectively superior product or outcome, maximizers are more willing than satisficers to choose an objectively inferior situation when it allows them to gain a relative advantage.

Table 2

Study 2: Positional choice over time: percentage of maximizers (subjects above the median of the sample on maximization score) and satisficers (subjects below the median of the sample on maximization score) choosing the positional option in each time category.

\begin{tabular}{llll}
\hline & Maximizer & Satisficer & Gap (Max - Sat) \\
\hline First six choice domains & 39 & 33 & 6 \\
Second six choice domains & 36 & 30 & 6 \\
Third six choice domains & 37 & 24 & 13 \\
Fourth six choice domains & 38 & 22 & 16 \\
Final five choice domains & 33 & 20 & 13 \\
\hline
\end{tabular}

This pattern was found across a broad spectrum of consumer choice domains, ranging from the luxuriousness of the car consumers wish to own to the quality of beer they would like to drink. These results call into question the "definitional" assumption made by previous work that maximizers' main interest is in achieving the best outcome in an objective sense (e.g., Schwartz et al., 2002) by showing that maximizers emphasize relative outcomes in their choices more than satisficers, even in situations where the absolute standard is clear.

Is an interest in relative standing an explicit part of the maximizer identity?

The results from study 1 paired with the overall choice data from study 2 demonstrate that maximizers are both more interested in relative standing than satisficers, and also that this interest can even override their desire for objective quality under some conditions. This suggests that the goal of relative standing is important to maximizers and part of their self concept. Analyses examining participants' choices over time go further to suggest that this interest in social comparison is actually an explicit self identity of maximizers. Specifically, while satisficers exhibited a decreasing tendency to choose positional options over time, suggesting that once the tradeoff became overt they may have realized that it is not socially desirable to sacrifice absolute standing to be better than others, maximizers' preference for positional options remained stable over time. This stability suggests that their interest in "winning" in a positional rather than an absolute sense is a way of viewing the self that they are comfortable with.

\section{Variation in choice domains}

Interestingly, while maximizers were more concerned with their relative position than satisficers overall, there was some variation across the domains. One factor that appears to be notable in explaining this variation has to do with the public visibility of the choice outcome. That is, in some of the choice domains, the positional disadvantage is naturally more likely to be highly visible and known to others (e.g., quality of car, sweater), while in other domains it is more likely to be hidden from public view and known only to the decision maker (e.g., frequency of sex per month, number of DVDs owned). The maximizer-satisficer difference appears to be larger in the visible domains than in the less visible ones. To investigate this quantitatively, two judges, blind to hypotheses, judged each scenario on the basis of how publicly visible the item in the scenario was. Results correlating the judges' average visibility ratings with the maximizer/satisficer gaps (i.e., the \% of maximizers choosing the positional option minus the \% of satisficers choosing the positional option) for each scenario showed a significant positive relationship, $r(27)=.37, p<.05$, such that the maximizer-satisficer difference was greater the more publicly visible the outcome of the choice was. While this pattern is suggestive, it is based on naturalistic scenarios that were not specifically designed to examine the public visibility issue. Studies 3 and 4 explore this possibility more systematically. 


\section{Overview: Studies 3 and 4}

The role of visibility is important from both a theoretical and practical standpoint. Visibility is important theoretically because it can increase our understanding of why maximizers are more influenced by social comparison than satisficers. Are maximizers looking to others because other people can provide information about what is the best in an objective sense or are maximizers looking to others to compete with them in an effort to be the best?

In studies 3 and 4 we disentangle these two possibilities. If maximizers' primary focus is on seeking information about what constitutes the best absolute outcome, then the public visibility of the outcome should not be relevant, and they should choose the same option in public as in private. In contrast, if maximizers' focus on social comparison is motivated by interpersonal competition and a desire to be the best in a relative sense, then they may be more likely to choose positionally when others will know about the decision outcome, but they may instead choose to maximize objective quality (even when it places them at a positional disadvantage) when the outcome is private. On the other hand, since past work indicates that satisficers are not interested in social comparison, we predicted that their choices should not vary as a function of the public visibility of the outcome.

Studies 3 and 4 examine this question using two different methodological approaches. Study 3 directly manipulates whether the positional advantage is visible to others or not and study 4 investigates the tradeoffs that maximizers and satisficers make for consumer products that are either typically consumed in public (e.g., MP3 player, sweater) or in private (e.g., pajamas, trash compactor) as well as examines implications for imitation products-naturally occurring "positional" choices of lower quality that are designed to have the outside appearance of high quality goods.

\section{Study 3: Manipulating choice outcome visibility}

Based on the results of study 2, we predicted that there would be an interaction between consumers' maximization scores and the visibility of the choice outcome on consumers' tendency to make positional versus absolute choices. When the positional advantage is visible to others, we predicted that maximizers would be more likely than satisficers to optimize social position, even when it means accepting a lower quality product. However, when the positional disadvantage is private and thus concealed from the public eye, we predicted that maximizers and satisficers would be equally likely to choose the objectively superior option.

\section{Method}

\section{Participants}

A total of 315 participants (44\% males) at a large, public university in the Midwest and a large, public university in the Southeast completed the study. Participants at the Midwestern university (University $1 ; N=181$ ) completed an online survey after being randomly emailed from the university directory. Those at the Southeastern university (University 2; $N=134$ ) participated as part of an extra credit assignment in their introduction to marketing course.

\section{Positional versus absolute choices}

Participants read instructions identical to those in study 2. After reading the instructions, the computer presented participants with the choice scenarios, one at a time. At University 1, the scenarios involved a necklace that varied in its value, an annual salary that was either higher or lower, and the quality of one's sex life, which varied on degree of satisfaction. At University 2, the scenarios involved a sweater that varied on the quality of its fabric and a pair of sunglasses that offered varying levels of UV protection. Each choice scenario offered an absolute option and a positional option, and participants chose the one they preferred (see Appendix B for examples).

\section{Public visibility}

To test whether the visibility of the decision outcome would influence participants' choices, a Public Visibility factor was manipulated between-subjects. Participants in the Visible condition were explicitly told that others would be aware of the outcome, while those in the Non-Visible condition were explicitly told that others would not become aware of the outcome. For example, the Visible (Non-Visible) condition for the sweater scenario read as follows:

You recently received a gift of a new sweater. Its value is in the fabric quality, so most people are able to tell its worth just by looking at it (so most people cannot tell its worth just by looking at it). Which would you prefer?

A Your sweater is a fair-quality brand. Nearly all your acquaintances have low-quality sweaters. [Selection indicative of positional choice]

B Your sweater is a medium-quality brand. Nearly all your acquaintances have real designer sweaters. [Selection indicative of absolute choice]

Visible condition participants saw publicly observable versions of all the choice domains; Non-Visible condition participants saw private versions of all the choice domains.

\section{Maximization scale}

Participants filled out the maximization scale $(\alpha=.61)$ (Schwartz et al., 2002), reported demographic information and were thanked and debriefed. The mean maximization score for students at University 1 was $4.33(\mathrm{SD}=4.45)$ and for students at University 2 it was $4.45(\mathrm{SD}=0.70)$.

\section{Design}

Study 3 was a Public Visibility (visible vs. non-visible) $\times$ Maximization Score (continuous factor ranging from 1 to 7 ) between-subjects design with choice (positional vs. absolute) as the dependent variable. 
Results

Composite variables representing the mean tendency to choose positionally across the choice domains were created for each university (absolute $=0$; positional $=1$ ). These composite scores as well as participants' continuous maximization scores were standardized within each university and then combined for analysis. There was no effect for university on any of the analyses, so this variable is not discussed further.

A regression was performed on positional choice with Maximization Score, a dummy variable for Visibility, and their interaction as the independent variables. As predicted, the results showed a significant two-way interaction between Maximization Score and Visibility $(\beta=.26, \quad t=2.35$, $p<.05)$. Also as predicted, simple effects analyses showed a positive association between an individual's score on the maximization scale and positional choice in the Visible condition $(\beta=.26, t=3.46, p<.01)$, but not in the Non-Visible condition $(\beta=-.004, t=-.05, p=.96)$. Fig. 1 depicts this interaction. Because the interaction graph shows a slight (nonpredicted) tendency for satisficers to choose more positionally in the non-visible than in the visible condition, we ran spotlight analyses to assess whether that unpredicted reversal was significant. Results showed that it was not. While maximizers $(+1 \mathrm{SD})$ made more positional choices when the outcome of their choice would be visible to others than when it would not, $t(311)=2.09, p<.05$, satisficers' ( $-1 \mathrm{SD}$ ) choices did not differ as a function of choice outcome visibility, $t(311)=-1.24, p>.20$.

\section{Discussion}

Study 3 replicates and extends the results of study 2 by demonstrating that, when social position and product quality

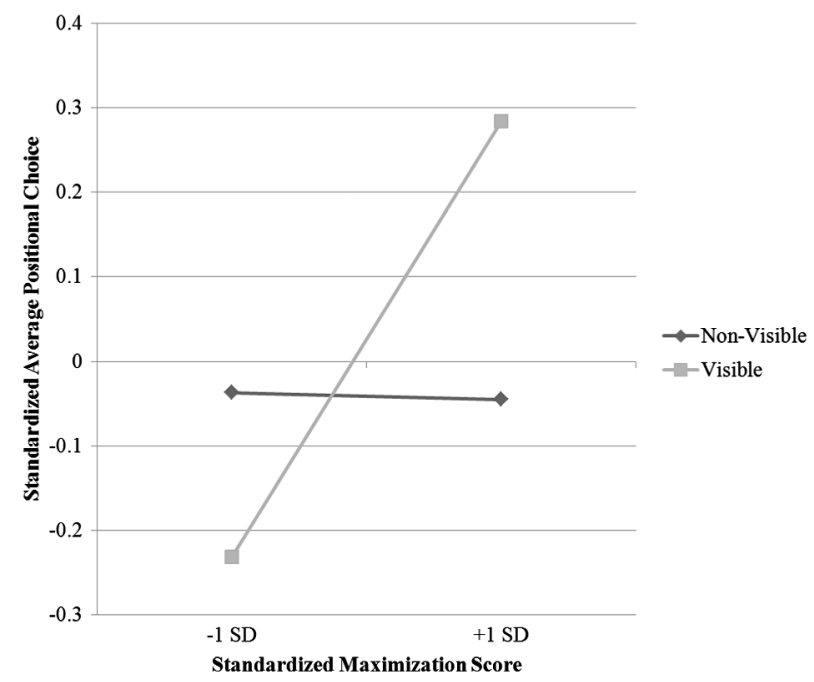

Fig. 1. Study 3: Regression lines for standardized average positional choice as a function of standardized maximization score in the visible and non-visible conditions. are pitted against each other in a publicly visible situation, maximizers place more weight on social position than product quality compared to satisficers. Taken by themselves, maximizers' public choices are compatible with the idea that they use social comparison information to learn about the absolute quality of outcomes, allowing them to determine what is the best outcome. If so, however, maximizers' choices should be independent of whether others can determine the quality of their possessions. Empirically, this is not the case as the results from the non-visible condition indicate. Instead, our results suggest that maximizers value a high relative standing more than high absolute quality and only maximize the latter when it does not threaten the former; hence, they attend to relative standards in public, but absolute standards in private. The choices of satisficers, on the other hand, are not affected by the publicity of the choice outcome. These observations add complexity to the past literature by showing that maximizers and satisficers as measured by the widely-used Schwartz et al. (2002) scale not only differ in the aspiration level they have for a given standard, as proposed by past work, but, in certain situations, they also appear to differ in the actual standards to which they aspire. In identifying an important moderator variable - the public visibility of the choice outcome - study 3 suggests that the same hard-driving and competitive nature that may drive maximizers' relentless pursuit of obtaining the best product may also make them interested in being the best in social competition situations.

\section{Study 4: Maximization and choices among publicly versus privately consumed products and imitation products}

Study 4 has two goals. First, it extends our investigation to a related but distinct contextual variable by examining the tradeoffs that maximizers and satisficers make between product quality and relative position for products that are typically consumed in public (e.g., brand of one's car) versus private (e.g., brand of one's pajamas). Based on the results from study 3 , we predicted that the public nature of a product's consumption would moderate the choices participants made, with maximizers being more willing than satisficers to choose a lower quality product that was paired with a better relative standing for publicly consumed products (e.g., MP3 player, sweater). We further predicted that maximizers and satisficers would be equally likely to emphasize product quality in their choices when the products are typically consumed in private and not visible to others (e.g., pajamas, trash compactor).

A second goal of study 4 was to investigate whether maximizers and satisficers differ in their preferences for fake products - products that are of inferior quality but actually appear from the outsider's perspective to be high quality goods. If maximizers place more weight on social standing than objective quality in publicly observable situations, they may express a preference for such imitation goods. In contrast, satisficers, who are generally not interested in social comparison, should see little appeal in imitation products. 


\section{Method}

\section{Participants}

One-hundred and three participants $(62 \%$ male $)$ from a large, public university in the Midwest completed this experiment online after being randomly selected from the student directory $(N=73)$ or as part of a subject pool requirement $(N=30)$.

\section{Positional versus absolute choices}

Participants read instructions that were identical to study 2 . Participants were then presented with 15 choice scenarios one at a time in a random order for each participant. The scenarios covered an array of consumer products (see Appendix C for examples). For each product, participants chose whether they would prefer an absolute or positional option. For example, the laptop scenario read as follows:

A.1 Your laptop was rated $60 / 100$ in quality by Consumer Reports. Nearly all your fellow students have low-quality laptops rated 50. [Selection indicative of a positional choice]

B Your laptop was rated $80 / 100$ in quality by Consumer Reports. Nearly all your fellow students have top-of-the-line laptops rated 95. [Selection indicative of an absolute choice]

The higher quality (but worse positionally) option was always described first and the positional option was always described second. Composite variables representing the mean tendency to choose positionally across the ten Visible choice domains and the five Non-Visible choice domains were created using the same procedure as in studies 2 and 3 (absolute choices $=0$; positional choices $=1$ ).

\section{Public versus private consumption}

Publicly consumed products were defined as those that many people other than the decision maker would see in everyday situations (Bearden \& Etzel, 1982; Childers \& Rao, 1992; Grewal, Mehta, \& Kardes, 2004). Ten of the 15 products were chosen because they met this criterion (e.g., sweater, purse). Privately consumed products were defined as those that not many people other than the decision maker would see on a daily basis. Five of the 15 products were chosen because they met this criterion (e.g., pajamas, trash compactor). The manipulation of public versus private products was done within-subjects, so all subjects saw both types of products.

\section{Fake versus non-fake products}

As discussed previously, some goods in the marketplace have inferior internal quality but have the outside appearance of higher quality goods. To investigate whether maximizers would be more likely than satisficers to choose an objectively inferior good if it looked like a higher quality good on the outside, an additional factor called Fake was manipulated between-subjects. For subjects in the Fake condition, the positional option was described as appearing to look like the higher quality item from the absolute option. For instance, the positional item for the laptop scenario for the Fake condition read as follows:

A.1 Your laptop was rated $60 / 100$ in quality by Consumer Reports, but has the outside casing of a top-of-the-line model (participants did not see the italics). Nearly all your fellow students have low-quality laptops rated 50 . [Selection indicative of a positional choice]

For subjects in the Non-Fake condition, the item indicative of positional choice was described without any reference to appearance (see A.1 above).

\section{Maximization scale}

After making their choices, participants completed the maximization scale $(\alpha=.67)$ (Schwartz et al., 2002), provided demographic information, and were thanked and debriefed. Participants' average maximization score in this sample was a $4.43(\mathrm{SD}=.76)$ and the median score in the sample was 4.38 .

\section{Manipulation check}

Study 4 included evaluations of both visible and non-visible products in the Fake and Non-Fake conditions. Since the Fake condition emphasizes appearance and makes it a salient feature (e.g., your mattress looks like a designer one), we wanted to confirm that it did not interact with participants' evaluations of the public and private products. A group of participants assigned to either the Fake or Non-Fake condition rated all the products on a seven point Likert scale ranging from $1=$ not visible at all—almost nobody would see it to 7 = highly visible — people would see it. Paired sample $t$-tests confirmed that visibility differences were of a similar magnitude in both the Fake $\left(M_{\text {non-visible }}=3.17\right.$ vs. $\left.M_{\text {visible }}=5.07, t(22)=6.23, p<.05\right)$ and Non-Fake conditions $\left(M_{\text {non-visible }}=2.92\right.$ vs. $M_{\text {visible }}=4.59$, $t(24)=5.01, p<.05)$.

Study 4 thus used a Product Visibility (publicly consumed products vs. privately consumed products) $\times$ Fake (fake vs. non-fake) $\times$ Maximization Score (continuous factor ranging from 1 to 7) design with Product Visibility a repeated measure and Fake a between-subjects factor. Our main prediction was that there would be two interactions, one between participants' maximization scores and the private versus public nature of the product's consumption and one between participants' maximization scores and whether a product was described as a fake or not.

\section{Results}

To test our main prediction, a repeated-measures ANCOVA was conducted using Fake as a between-subjects factor, Product Visibility as a within-subjects factor, and Maximization Score as a continuous measured factor and participants' mean tendency to choose positionally as the dependent variable. The model included all possible interactions of the variables (i.e., within, between, and covariate). Analyses revealed significant effects of Maximization Score, $F(1,99)=4.9, p<.05$, Product Visibility, $F(1,99)=4.5, p<.05$ and Fake, $F(1,99)=4.4, p<.05$ as well 
as a nonsignificant three way interaction between them $(F<1)$. Consistent with our main hypothesis, there were also significant Product Visibility $\times$ Maximization Score, $F(1,99)=8.8$, $p<.01$ and Fake $\times$ Maximization Score interactions, $F(1$, 99) $=6.7, p<.05$.

To explore the Product Visibility $\times$ Maximization Score interaction, we examined the simple effect of Maximization Score on positional choice in both the Visible and Non-Visible conditions. As predicted, there was a positive association between an individual's score on the maximization scale and their tendency to make a positional choice in the Visible condition $(\beta=.09, t=3.91, p<.01)$, but not in the Non-Visible condition $(\beta=.008, t=.34, p=.73)$. Fig. 2 visually depicts this interaction.

To explore the significant Fake $\times$ Maximization Score interaction, we examined the simple effect of Maximization Score on positional choice in both the Fake and Non-Fake conditions. As predicted, there was a positive association between an individual's score on the maximization scale and their propensity to make a positional choice in the Fake condition $(\beta=.11, t=3.98, p<.01)$, but not in the Non-Fake condition $(\beta=.006, t=.19, p=.85)$. Fig. 3 visually depicts this interaction.

\section{Discussion}

By demonstrating that maximizers' choices are influenced by the public versus private nature of the product category and the manipulated fake variable but satisficers' choices are not, study 4 adds significantly to our understanding of how maximizers weigh objective quality versus social standing. Rather than looking to others for information about what is the best product, in contexts where social rivalry is highlighted, maximizers instead appear to be looking to others for

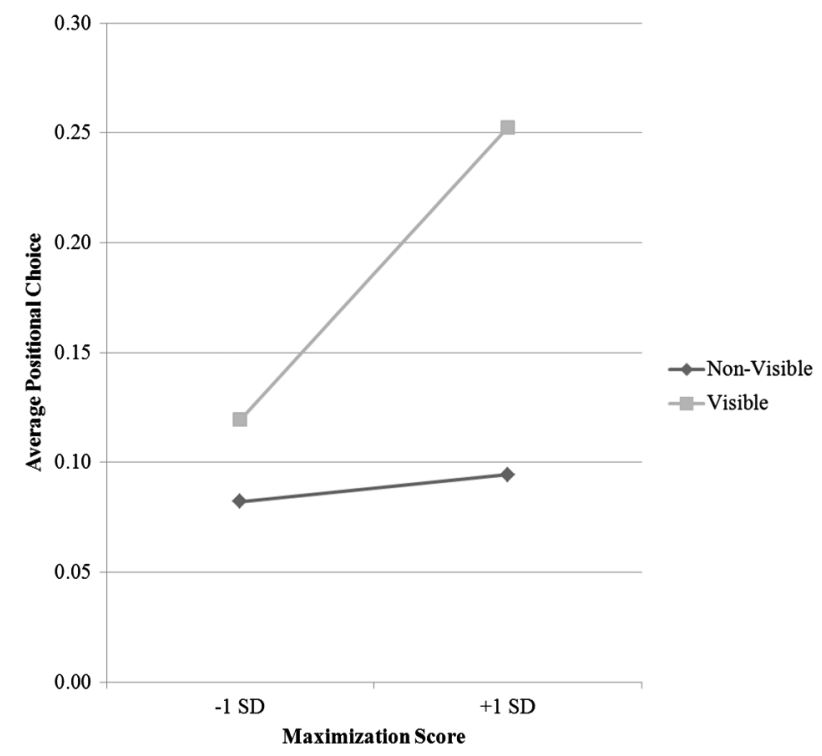

Fig. 2. Study 3: Regression lines for average positional choice as a function of maximization score in the visible and non-visible conditions.

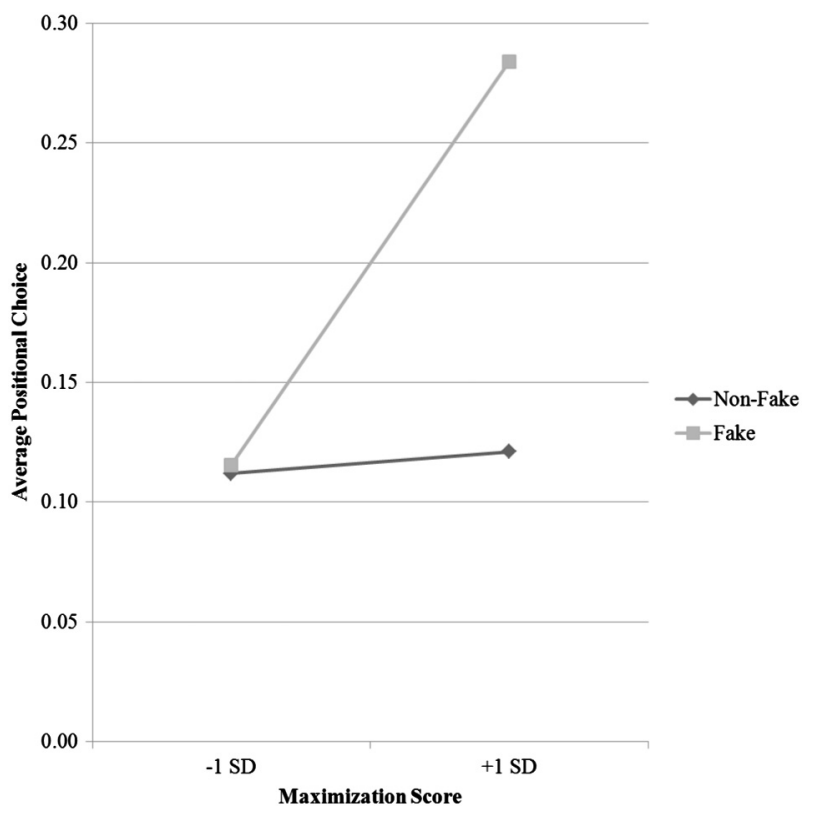

Fig. 3. Study 4: Regression lines for average positional choice as a function of maximization score in the fake and non-fake conditions.

information on who has the best product. In line with their focus on wanting to appear as the best, more maximizers than satisficers preferred imitation products. Simply giving lower quality products the external appearance of high quality items was sufficient to make them more attractive to maximizers. Satisficers, in contrast, were uninterested in products giving merely an external appearance of high quality.

\section{General discussion}

\section{Summary and theoretical implications}

The current studies build a theoretical bridge between two literatures, one on maximizing and satisficing in decision making and the other on relative and absolute choices. Past work has identified two types of consumers on the basis of how much they try to optimize in their decision making (Schwartz et al., 2002). Maximizers are consumers who are exhaustive in their search for the best product, while satisficers tend to truncate the search process as soon as they have identified a product that meets a minimum threshold of acceptability (Schwartz et al., 2002). Previous work investigating individual differences in maximizing and satisficing has operated under the implicit assumption that there is a single "definitional" goal driving maximizers' behavior-i.e., that they have a relentless drive to obtain the best products in an objective sense (e.g., Schwartz et al., 2002). Building upon this past work our results uncover, for the first time, that the maximizer identity also includes an additional — and sometimes conflicting — goal, that of a desire to be the best in social competition situations. When presented with tradeoffs that forced participants to make a choice between these two motivations maximizers were actually more willing than satisficers to give up objective 
quality in order to get a higher social standing than were satisficers. Further analyses investigating maximizers' and satisficers' choices over time suggested that maximizers' interest in social standing is explicit and conscious rather than driven by an implicit motivation that operates below their awareness.

The current work also investigates the processes that underlie maximizers' relative preference for positional standing over objective quality, showing that it is moderated by whether the outcome of their choice would be known to others or not. Specifically, product choice has few implications for the perception of the owner by others when the outcome of the decision is not apparent to observers. Under these low visibility conditions, we found that participants' maximization scores did not influence their choices. In contrast, when the product has high public visibility and its quality is apparent to others, we found that maximizers and satisficers differed in the choices made. In this case, maximizers preferred the option that increases their relative standing vis a vis others more than satisficers, even when that choice implied the acquisition of an inferior product. This moderating role of product visibility emerged across three separate but convergent methodologies - when product visibility was experimentally manipulated by informing consumers that others either would or would not know about the positional advantage (study 3); when product visibility was varied naturalistically by using products that naturally vary in the public nature of their consumption (study 4); and when choices were about imitation products, those that are lower quality but have the outside appearance of higher quality products (study 4).

The observation that maximizers, as measured by the Schwartz et al. (2002) scale, focus on their positional standing even at the expense of product quality would seem to suggest an inconsistency in the scale relative to what would be predicted by the standard economic model and what would be suggested by Simon's (1956) original conceptualization of the optimizing and satisficing constructs. That is, Simon's seminal insight was that cognitive and situational constraints prevent decision makers from optimizing ("maximizing") their choices. In proposing this concept of bounded rationality, Simon was agnostic about what decision makers were trying to maximize. Still, implicit in both Simon's work (1955) as well as work on the maximizer/satisficer individual differences distinction is the notion that "maximizers" and "satisficers" aspire to the same standard and vary only in their aspiration level for that standard, with maximizers aspiring to a higher level and satisficers to a comparatively lower level. In contrast, the current results suggest that maximizers and satisficers, as operationalized by the Schwartz et al. (2002) scale, not only differ in their aspiration levels but also differ in their conceptualization of what the "best" means. This discovery adds complexity to the current literature and highlights differences in the conceptualization of maximizing and satisficing as operationalized by the Schwartz et al. (2002) scale and the classical concepts of "maximizers" and "satisficers."

There are two possible reasons why maximizers may favor relative over absolute outcomes more than satisficers when the two motivations are juxtaposed. On the one hand, maximizers, people who dispositionally go to great lengths to secure the best in life, are likely driven, competitive individuals by nature and thus may have as part of their self concepts the tendency to seek both types of "bests." This means that when quality is salient, they maximize on that dimension and when relative standing is salient, they maximize on it instead. Their competitive nature furthermore may lead them to prefer relative standing when the two motivations are juxtaposed (unless the situation is private and no one will know about their superiority). Another possibility is that maximizers may be more sensitive than satisficers to the standards of others. Perhaps situations that prime an audience (e.g., such as the "public" conditions of studies 3 and 4) prime maximizers to imagine themselves in the eyes of those others, raising the salience of the goal of winning or being the best. Although definitively distinguishing between these two possibilities is beyond the scope of the current paper, each suggests that there are as of yet unmeasured constructs included in the maximization scale above and beyond a simple propensity to search for the "best" item in an objective sense. Future research should investigate which of these underlying processes underlies maximizers' interest in relative standing.

The current results also provide a new framework through which to view previous empirical findings. Past work has shown that maximizers are more interested in social comparison than satisficers (Schwartz et al., 2002). In light of this finding, past work surmised that social comparison information is useful to maximizers because it provides a "social reality test" (Festinger, 1950) - it helps them to cut through an overwhelming array of options to gain information about what is the best product in an absolute sense in situations where the best is ambiguous from the context (e.g., Iyengar et al., 2006; Schwartz et al., 2002). The current research suggests instead that rather than using social comparison for information, "being the best" may be a goal in and of itself for maximizers in certain situations.

\section{Limitations and future directions}

The current studies used participants' choices of absolute and positional products as well as their patterns of choices over time as a way of inferring the importance of social position as an identity process for maximizers. While this is an important first step, future research should address limitations of this approach. For instance, while the current studies investigate the maximizer construct in terms of a widely-used individual difference scale, identity-based motivation theory (Oyserman, 2007, 2009; Oyserman, Fryberg, \& Yoder, 2007) additionally predicts that the meaning of an identity is dynamically constructed in context. This raises the interesting question of whether situational manipulations, such as primes, of the maximizer or satisficer identities would produce similar results as those shown here. For instance, one could present participants with survey items (e.g., "Getting the best is the most important thing in life, otherwise why bother to search at all?; Sometimes it is silly to run around looking for a better option, because what you have is good enough") along with a manipulation of the response scale to induce people to endorse 
one goal over another. To the degree that the maximizer construct as measured in the Schwartz et al. (2002) scale is similar to the maximizer construct as conceptualized, one would expect such a prime to lead to a similar pattern of results as found here. Another interesting possibility along these lines would be to investigate whether priming the two maximizing goals of absolute quality versus relative position would in turn activate the concepts of maximizing and satisficing themselves. For instance, to the degree that an interest in relative position is a component of the maximizer identity, then focusing attention on that goal as in a print advertisement (e.g., "This is the dress that will make you stand out at the party") or in a product display that highlights the exclusive nature of the product should increase the degree to which people endorse a maximizer identity. On the other hand, focusing on lack of search and "good enough" as in an advertisement (e.g., "Why search any further? This refrigerator never breaks") or a more utilitarian "something for everyone" product display format should increase the salience of a satisficer identity.

An additional limitation of the current approach is that it uses a single scale to focus on both maximizing and satisficing, two potentially only weakly related identities, with low maximization acting as a "stand in" for the satisficing construct. Future work that attempts to prime or measure each construct separately, as described above, could make it possible to more clearly test the relative contribution of each to consumer choice. Doing so can also provide an opportunity for a test of the cultural universality of the maximizer identity. For instance, is maximizers' interest in relative standing a goal that is particularly prevalent in individualistic cultures, or is their focus on social comparison universal and also present within a collectivist frame? Examining the maximizing and satisficing constructs cross-culturally can provide information as to the universality and composition of these identities.

It may also be interesting to investigate the degree to which maximizers' choices serve a functional role (e.g., Katz, 1960; Shavitt, 1989). The fact that they prefer relative standing in public more than satisficers, even when that standing comes from counterfeit products, raises the possibility that maximizers may be using their choices in either a social-adjustive or a value-expressive manner, perhaps as a way to signal their status or desire to be the best to others (Gino, Norton, \& Ariely, 2010; Wilcox, Kim, \& Sen, 2009). It would be interesting to distinguish between these two possible functional roles in maximizers' choice behavior not only because it may provide theoretical insight but also because recent work suggests that usage of counterfeit products can have negative psychological ramifications (e.g., Gino et al., 2010). While the focus here was on the maximization construct in general, future research may also benefit from investigating the different aspects of maximization to assess which, if any, are more correlated with maximizers' pursuit of relative standing and absolute quality. Past work has indicated that there are distinct cognitive, emotional, and behavioral aspects of maximization (Nenkov et al., 2008). An interesting topic for future research thus may be to examine the correlation between these constructs and consumers' tendency toward positional choice.

It is interesting to note that the current paper as well as past work on the maximizing and satisficing constructs has focused to a large degree on the motivations of maximizers and has paid less attention to the underlying motivations of satisficers. One reason maximizers may capture a larger portion of the research spotlight is because that side of the spectrum seems to be where "the action is." Maximizers work harder and tend to do better by objective metrics, but, at the same time, maximizing has been shown to have negative psychological ramifications. Satisficers, on the other hand, appear to be better adjusted with higher levels of well-being. For this reason, future research should be directed toward investigating the decision making style of satisficers as it may be useful in uncovering aspects of decision making that may lead to positive rather than negative psychological outcomes.

In most of the choice situations we encounter in daily life, quality and relative standing are confounded (e.g., the best wine is also often the most expensive; the highest quality diamond is also the most rare). This means that when consumers choose a high quality item, the reason behind their choice is almost always ambiguous-did they choose it because they are seeking quality per se, or was the choice motivated by the status that comes with the object? To avoid this ambiguity, the current studies purposefully used tradeoffs that separated out these two motivations in order to obtain a deeper theoretical understanding of what drives maximizers' interest in social information.

\section{The darker side of maximizing: Reducing the appeal of counterfeit products}

The current studies also bring to light one of the darker sides of maximizers' preference for positional goods - their possible willingness to consume fake or counterfeit products that have the outside appearance of prestige goods. While some imitation products are legal, other imitation goods such as counterfeit luxury items are not and cost retailers millions of dollars a year in lost sales and diluted brand image (Commuri, 2009; Wilcox et al., 2009). One intuitive response is to discourage counterfeit purchases by using the low quality of the products themselves as a deterrent. However, the fake condition of study 4 suggests that the consumers who are most likely to be interested in counterfeit items - maximizers - are careful decision makers who have likely already made a mindful choice to sacrifice product quality in favor of the outside appearance of positional standing. Instead, the current results suggest a different strategy. Brands could create messages that present counterfeit apparel as the opposite of a status signal. For instance, using a message that highlights the fact that everyday consumers can often "see through" counterfeit purchases and may judge the owner of one as someone who is a "fake" should be more effective at dissuading maximizers from such purchases as it would create the possibility that such a purchase may have the opposite effect on their image than the one they desired. 


\section{Conclusions}

The current studies show that in addition to "positional goods" there may be an identifiable group of "positional individuals" (maximizers) who are willing to sacrifice objective quality for relative standing in a wide variety of situations. Indeed, the fact that maximizers-decision makers whose primary concern is in obtaining "the best" - are repeatedly choosing objectively inferior products and outcomes is in some sense ironic. However, choosing the worst in order to be the best helps to illuminate the larger and more general conclusion that the current paper has brought to light, namely that the maximizer self concept is more complicated than has been previously assumed and includes not only an aspect that focuses on product quality in an objective sense but also a facet that emphasizes social status and social competition. This insight not only contributes to our practical marketing knowledge by suggesting novel product positioning combinations but also increases our theoretical understanding of the relationship between individual differences in maximization and the classical decision making concepts of maximizing and satisficing.

\section{Appendix A. Study 1: Choice Tradeoffs}

\section{A.1. Luxuriousness of your car}

- Your car rates 5 out of 10 points on a luxury scale; others' cars rate 3 out of 10 .

- Your car rates 7 out of 10 points on a luxury scale; others' cars rate 9 out of 10 .

\section{A.2. Quality of your car}

- Your car is a fair quality brand rated $6 / 10$ on parts and performance by Automotive Weekly. Nearly all of your acquaintances' cars are low-quality models rated 4/10.

- Your car is a medium-quality brand rated 7/10 on parts and performance by Automotive Weekly. Nearly all your acquaintances' cars are luxury models rated 9/10.

\section{A.3. Quality of your sweater}

- Your sweater is a fair-quality brand. Nearly all your acquaintances have low-quality sweaters.

- Your sweater is a medium-quality brand. Nearly all your acquaintances have real designer sweaters.

\section{A.4. Number of job offers}

- You receive three job offers while others receive two job offers.

- You receive five job offers while others receive seven job offers.

\section{A.5. Level of your personal education}

- You have 12 years of education (high school); others have 8.

- You have 16 years of education (college); others have 20 (graduate degree).

\section{A.6. Yearly income}

- Your current yearly income is $\$ 50,000$; others earn $\$ 25,000$.

- Your current yearly income is $\$ 100,000$; others earn $\$ 200,000$.

\section{A.7. Chance of getting a job two months after graduation}

- You have a 50\% chance of getting a job within 2 months after graduation, others have a $40 \%$ chance.

- You have a $60 \%$ chance of getting a job within 2 months after graduation, others have a $75 \%$ chance.

\section{A.8. Number of invites to formals}

- You were asked to formals by 2 people and your friends were asked by 1 .

- You were asked to formals by 3 people and your friends were asked by 5 .

\section{A.9. Personal physical shape}

- You are slightly out of shape, others are very out of shape.

- You are in good shape, others are in excellent shape.

\section{A.10. Sound quality of your MP3 player}

- Your MP3 player has fair sound quality. Nearly all your acquaintances have only portable CD players.

- Your MP3 player is a generic brand with medium sound quality. Nearly all your acquaintances' MP3 players are real iPods with high sound quality.

\section{A.11. Time spent studying for test}

- You spend 20 hours a studying for a test; other people spend 25 hours.

- You spend 15 hours a studying for a test; other people spend 10 hours.

\section{A.12. Quality of beer you are drinking}

- Your beer is a 4 out of 10 in quality, others' beer is a 2 out of 10 .

- Your beer is a 6 out of 10 in quality, others' beer is a 8 out of 10 . 


\section{A.13. Number of days you are in Florida for spring break}

- You are going to Florida for three days during spring break while others can only go for two days.

- You are going to Florida for five days during spring break while others can go for a whole week.

\section{A.14. Protection of your sunglasses}

- Your sunglasses provide fair UV protection. Nearly all your friends have very low-quality sunglasses that provide poor UV protection.

- Your sunglasses are generic brand with medium-level UV protection. Nearly all your friends have real designer sunglasses with high UV protection.

\section{A.15. Frequency of sex per month}

- You have sex 5 times per month; others have sex 2 times a month.

- You have sex 8 times per month; others have sex 12 times a month.

\section{A.16. Time spent training for athletics}

- You spend 150 hours training for an athletic competition; other people spend 200 hours.

- You spend 100 hours training for an athletic competition; other people spend 50 hours.

\section{A.17. Number of bids received for a fraternity/sorority}

- You received a bid from 1 sorority and your friends received bids from 0 .

- You received bids from 3 sororities and your friends received bids from 4 .

\section{A.18. Number of invites to parties}

- You were invited to two parties on Friday night; others were invited to one.

- You were invited to four parties on Friday night; others were invited to six.

\section{A.19. Frequency of going to movies}

- You go to the movies 2 times a month; others go 1 time a month.

- You go to the movies 4 times a month; others go 6 times a month.

\section{A.20. Quality of suit for interview}

- You buy a suit for an interview. The quality is 6 out of 10 , others applying have suits that are 5 out of 10 .

- You buy a suit for an interview. The quality is 8 out of 10 , others applying have suits that are 9 out of 10 .

\section{A.21. Number of DVDs owned}

- -You have 30 DVDs; others have 20.

- -You have 45 DVDs; others have 60.

\section{A.22. What row your football seats are in}

- You have 40th row seats at the [school name] football game and nearly all your friends and acquaintances have 65th row.

- You have 30th row seats at the [school name] football game; nearly all your friends and acquaintances have 5 th row.

\section{A.23. Quality of spring break destination}

- You are going to New York for spring break, others are going to Virginia Beach.

- You are going to Florida for spring break, others are going to Cancun.

\section{A.24. Grades on exams}

- You get Bs and Cs on your exams while others get all Cs.

- You get all As except one B on your exams while others get straight As.

\section{A.25. Poker winnings}

- You win \$20 in a poker game; others win \$10.

- You win $\$ 50$ in a poker game; others win $\$ 100$.

\section{A.26. Number of Facebook friends}

- You have 140 friends on Facebook and your peers have 90.

- You have 200 friends on Facebook and your peers have 250 .

\section{A.27. Number of rooms in house}

- Your home has five rooms; other people's homes have three rooms.

- Your home has seven rooms; other people's homes have ten rooms.

\section{A.28. Frequency of eating out per month}

- You eat out at a nice restaurant 4 times per month; others eat out once a month.

- You eat out at a nice restaurant 8 times per month; others eat out 12 times per month.

\section{A.29. Frequency of going out per week}

- You go out 3 times a week; others go out 2 times a week.

- You go out 4 times a week; others go out 5 times a week. 


\section{Appendix B. Study 3: Choice Tradeoffs and Visibility Manipulations}

\section{B.1. Sweater: Visible (Non-visible)}

You recently received a gift of a new sweater. Its value is in the fabric quality, so most people are able to tell its worth just by looking at it (so most people cannot tell its worth just by looking at it). Which would you prefer?

- Your sweater is a fair-quality brand. Nearly all your acquaintances have low-quality sweaters.

- Your sweater is a medium-quality brand. Nearly all your acquaintances have real designer sweaters.

\section{B.2. Necklace: Visible (Non-visible)}

You recently received a gift of a new necklace. Its value is very obvious and noticeable to experts and non-experts alike, so most people can tell (its value is subtle and only noticeable to experts, so people cannot tell).

- Your necklace is worth $\$ 5,000$ and your friends are wearing ones worth about $\$ 3,000$ each.

- Your necklace is worth $\$ 6,000$ and your friends are wearing ones worth about $\$ 8,000$ each.

\section{B.3. Sunglasses: Visible (Non-visible)}

An innovation in sunglass lens development has been established such that the color of the lens indicates the level of UV protection of the glasses (such that a dot on the sunglass frame indicates the level of UV protection of the glasses). So, everyone knows the UV protection of others' sunglasses. (So, no one can tell the UV protection of others' sunglasses.) Which would you prefer?

- Your sunglasses provide fair UV protection. Nearly all your friends have very low-quality sunglasses that provide poor UV protection.

- Your sunglasses are generic brand with medium-level UV protection. Nearly all your friends have real designer sunglasses with high UV protection.

\section{B.4. Salary: Visible (Non-visible)}

At your firm, salary information is publicly posted at the end of the fiscal year (kept very private). So, employees know (do not know) each other's salaries.

- You make $\$ 50,000$ per year and nearly all of your co-workers make $\$ 30,000$.

- You make $\$ 60,000$ per year and nearly all of your co-workers make $\$ 80,000$.

\section{B.5. Sex Life: Visible (Non-visible)}

In your group of friends and acquaintances, information about each other's sex lives is a frequent topic of discussion (is almost never discussed). So, everybody (nobody) knows about each other's sex lives.

- You are fairly satisfied with your sex life and nearly all your friends have less exciting sex lives.

- You are satisfied with your sex life and nearly all your friends have more exciting sex lives.

\section{Appendix C. Study 4: Choice Tradeoffs and Manipulations}

\section{C.1. Visible Choice Domains}

\section{C.1.1. Sweater: Non-Fake (Fake)}

- Your sweater is a fair-quality brand (but looks identical to a real designer one). Nearly all your acquaintances have low-quality sweaters.

- Your sweater is a medium-quality brand. Nearly all your acquaintances have real designer sweaters.

\section{C.1.2. Purse: Non-Fake (Fake)}

- Your purse is fair quality (but looks identical to a high-quality designer brand (e.g., Louis Vuitton)). Almost all your acquaintances' purses are low-quality brands.

- Your purse is a medium-quality, non-designer brand. Almost all of your acquaintances' purses are high-quality designer brands (e.g., Louis Vuitton).

\section{C.1.3. Sunglasses: Non-Fake (Fake)}

- Your sunglasses (look identical to real designer ones but provide only) provide fair UV protection. Nearly all your friends have very low-quality sunglasses that provide poor UV protection.

- Your sunglasses are generic brand with medium-level UV protection. Nearly all your friends have real designer sunglasses with high UV protection.

\section{C.1.4. Shoes: Non-Fake (Fake)}

- Your shoes are rated 5 out of 10 in comfort (but look exactly like designer brand shoes rated 10). Almost all of your acquaintances' shoes are rated 3 .

- Your shoes are rated 7 out of 10 in comfort. Almost all of your acquaintances' shoes are designer brands rated 10 .

\section{C.1.5. Car: Non-Fake (Fake)}

- Your car is a fair quality brand rated $6 / 10$ on parts and performance by Automotive Weekly (but it has the identical outside appearance of a well known luxury model rated 9). 
Nearly all of your acquaintances' cars are low-quality models rated $4 / 10$

- Your car is a medium-quality brand rated 7/10 on parts and performance by Automotive Weekly. Nearly all your acquaintances' cars are luxury models rated 9/10.

\section{C.1.6. Cell Phone: Non-Fake (Fake)}

- Your cell phone is fair quality (but its outside appearance is indistinguishable from a brand name, high-quality model). Nearly all of your acquaintances have low-quality, second hand phones.

- Your cell phone is a medium-quality model. Nearly all your acquaintances' cell phones are brand name, high-quality models.

\section{C.1.7. Running Shoes: Non-Fake (Fake)}

- Your running shoes are only fair performance (but look identical to high performance ones). Nearly all your teammates' shoes are low-performance non-brand name.

- Your running shoes are medium performance. Nearly all your teammates' shoes are high-performance, brand name ones.

\section{C.1.8. Backpack: Non-Fake (Fake)}

- Your backpack (looks identical to a genuine top-of-the-line backpack but is actually) is a generic brand rated 60/100 on durability. All your friends have very low-quality backpacks rated 50 .

- Your backpack is a generic brand rated $80 / 100$ on durability. All your friends have top-of-the-line backpacks rated 95 .

\section{C.1.9. MP3 Player: Non-Fake (Fake)}

- Your MP3 player (is a knock off that looks identical to an iPod but has only) has fair sound quality. Nearly all your acquaintances have only portable CD players.

- Your MP3 player is a generic brand with medium sound quality. Nearly all your "acquaintances" MP3 players are real iPods with high sound quality.

\section{C.1.10. Laptop: Non-Fake (Fake)}

- Your laptop was rated $60 / 100$ in quality by Consumer Reports (but it has the outside casing of a top-of-the-line model). Nearly all your fellow students have low-quality laptops rated 50.

- Your laptop was rated $80 / 100$ in quality by Consumer Reports. Nearly all your fellow students have top-of-the-line laptops rated 95 .

\section{C.2. Non-visible Choice Domains}

\section{C.2.1. Blanket: Non-Fake (Fake)}

- Your blanket is fair quality (but looks exactly like a high-quality imported one). Almost all your friends' blankets are poor quality.

- Your blanket is medium-quality. Almost all your friends' blankets are high-quality imported models.

\section{C.2.2. Pajamas: Non-Fake (Fake)}

- Your pajamas are a fair quality, generic brand rated 6/10 in comfort (but look identical to real designer pajamas). Nearly all your friends have low quality pajamas rated $3 / 10$ in comfort.

- Your pajamas are a medium-quality, generic brand rated $7 / 10$ in comfort. Nearly all your friends have real designer pajama rated $10 / 10$.

\section{C.2.3. Ice Maker: Non-Fake (Fake)}

- Your ice maker was rated 5 out of 10 in quality (but its performance is indistinguishable from a designer one that was rated 10). Most of your acquaintances have low quality ice makers rated 3 .

- Your ice maker was rated 7 out of 10 in quality. Most of your acquaintances have designer ice makers rated 10 .

\section{C.2.4. Mattress: Non-Fake (Fake)}

- Your mattress was rated 5 out of 10 in comfort (but looks exactly like a designer one that was rated 10). Almost all your acquaintances have mattresses rated 3 .

- Your mattress was rated 7 out of 10 in comfort. Almost all your friends have designer mattresses rated 10 .

\section{C.2.5. Trash Compactor: Non-Fake (Fake)}

- Your trash compactor is fair quality (but looks identical to a high quality one). Your acquaintances all have low-quality trash compactors.

- Your trash compactor is medium quality. Your acquaintances all have high-quality trash compactors.

\section{References}

Bearden, W. O., \& Etzel, M. J. (1982). Reference group influence on product and brand purchase decisions. Journal of Consumer Research, 9, 183-194, http://dx.doi.org/10.1086/208911.

Childers, T. L., \& Rao, A. R. (1992). The influence of familial and peer-based reference groups on consumer decisions. Journal of Consumer Research, 19(2), 198-211, http://dx.doi.org/10.1086/209296.

Chowdhury, T. G., Ratneshwar, S., \& Mohanty, P. (2009). The time-harried shopper: Exploring the differences between maximizers and satisficers. Marketing Letters, 20(2), 155-167, http://dx.doi.org/10.1007/s11002-0089063-0. 
Commuri, S. (2009). The impact of counterfeiting on genuine-item consumers' brand relationships. Journal of Marketing, 73(3), 86-98, http://dx.doi.org/ 10.1509/jmkg.73.3.86.

Dar-Nimrod, I., Rawn, C. D., Lehman, D. R., \& Schwartz, B. (2009). The maximization paradox: The costs of seeking alternatives. Personality and Individual Differences, 46(5-6), 631-635, http://dx.doi.org/10.1016/j.paid. 2009.01.007.

de Bruin, W. B., Parker, A. M., \& Fischhoff, B. (2007). Individual differences in adult decision-making competence. Journal of Personality and Social Psychology, 92(5), 938-956, http://dx.doi.org/10.1037/0022-3514.92.5. 938.

Easterlin, R. A. (1974). Does economic growth improve the human lot? Some empirical evidence. In P. A. David, \& M. W. Reder (Eds.), Nations and households in economic growth: Essays in honor of Moses Abramowitz (pp. 89-125). New York: Academic Press.

Gino, F., Norton, M. I., \& Ariely, D. (2010). The counterfeit self: The deceptive costs of faking it. Psychological Science, 21(5), 712-720, http://dx.doi.org/ 10.1177/0956797610366545.

Grewal, R., Mehta, R., \& Kardes, F. R. (2004). The timing of repeat purchases of consumer durable goods: The role of functional bases of consumer attitudes. Journal of Marketing Research, 41(1), 101-115, http://dx.doi.org/ 10.1509/jmkr.41.1.101.25090.

Huguet, P., Dumas, F., Marsh, H., Régner, I., Wheeler, L., Suls, J., et al. (2009). Clarifying the role of social comparison in the big-fish-little-pond effect (BFLPE): An integrative study. Journal of Personality and Social Psychology, 97(1), 156-170, http://dx.doi.org/10.1037/a0015558.

Iyengar, S. S., Wells, R. E., \& Schwartz, B. (2006). Doing better but feeling worse: Looking for the "best" job undermines satisfaction. Psychological Science, 17(2), 143-150, http://dx.doi.org/10.1111/j.1467-9280.2006.01677.x.

Katz, D. (1960). The functional approach to the study of attitudes. Public Opinion Quarterly, 24(2), 163-204.

Nenkov, G. Y., Morrin, M., Ward, A., Schwartz, B., \& Hulland, J. (2008). A short form of the maximization scale: Factor structure, reliability and validity studies. Judgment and Decision Making, 3(5), 371-388.

Oyserman, D. (2007). Social identity and self-regulation. In A. Kruglanski, \& T. Higgins (Eds.), Handbook of social psychology (pp. 432-453) (2nd ed.). NY: Guilford Press.

Oyserman, D. (2009). Identity-based motivation: Implications for actionreadiness, procedural-readiness, and consumer behavior. Journal of Consumer Psychology, 19, 250-260.
Oyserman, D., Fryberg, S., \& Yoder, N. (2007). Identity-based motivation and health. Journal of Personality and Social Psychology, 93, 1011-1027.

Parker, A. M., de Bruin, W. B., \& Fischhoff, B. (2007). Maximizers versus satisficers: Decision-making styles, competence, and outcomes. Judgment and Decision Making, 2(6), 342-350.

Polman, E. (2010). Why are maximizers less happy than satisficers? because they maximize positive and negative outcomes. Journal of Behavioral Decision Making, 23(2), 179-190, http://dx.doi.org/10.1002/bdm.647.

Schwartz, B. (2004). The paradox of choice: Why more is less. New York: Harper Collins.

Schwartz, B., Ward, A., Monterosso, J., Lyubomirsky, S., White, K., \& Lehman, D. R. (2002). Maximizing versus satisficing: Happiness is a matter of choice. Journal of Personality and Social Psychology, 83(5), 1178-1197, http://dx.doi.org/10.1037/0022-3514.83.5.1178.

Shavitt, S. (1989). Operationalizing functional theories of attitude. In A. R. Pratkanis, S. J. Breckler, \& A. G. Greenwald (Eds.), Attitude structure and function (pp. 311-337). Hillsdale, NJ: Erlbaum.

Simon, H. (1956). Rational choice and the structure of the environment. Psychological Review, 63(2), 129-138.

Solnick, S. J., \& Hemenway, D. (2005). Are positional concerns stronger in some domains than in others? American Economic Review, 95(2), 147-151, http://dx.doi.org/10.1257/000282805774669925.

Swan, J. E. (1969). Experimental analysis of predecision information seeking. Journal of Marketing Research, 6(2), 192-197, http://dx.doi.org/10.2307/ 3149671 .

Wilcox, K., Kim, H. M., \& Sen, S. (2009). Why do consumers buy counterfeit luxury brands? Journal of Marketing Research, 46(2), 247-259, http://dx. doi.org/10.1509/jmkr.46.2.247.

Wright, P. (1975). Consumer choice strategies: Simplifying vs. optimizing. Journal of Marketing Research, 12(1), 60-67, http://dx.doi.org/10.2307/ 3150659.

Zeelenberg, M., \& Pieters, R. (2007). A theory of regret regulation 1.0. Journal of Consumer Psychology, 17(1), 3-18, http://dx.doi.org/10.1207/ s15327663jcp1701_3.

Zhou, R., \& Soman, D. (2003). Looking back: Exploring the psychology of queuing and the effect of the number of people behind. Journal of Consumer Research, 29(4), 517-530. 